\title{
Desarrollo de la creatividad: Diseño y evaluación de un programa de intervención
}

Pedro Allueva

Universidad de Zaragoza

Zaragoza, España

Se aplicó el programa de intervención, desarrollado en base a las cinco etapas del proceso creativo de Amabile a un grupo de estudiantes de segundo curso de la carrera de diseño industrial, teniendo en cuenta la combinación de atributos intelectuales, el estilo cognitivo, la personalidad y las estrategias. Se procuró eliminar las barreras a la creatividad, crear un clima adecuado y estimular actitudes favorables que ayudaran a desarrollar habilidades creativas. Los resultados obtenidos demuestran que la implementación de programas de creatividad en las escuelas, adaptados a los diferentes niveles, puede producir incrementos significativos en la capacidad creativa.

creatividad / programa de intervención

\section{Development of creativity: design and evaluation of a program of intervention}

The intervention program based on the five stages of the Amabile's creative process was applied to a group of second year students majoring in industrial design considering the combination of intellectual attributes, cognitive style, personality and strategies. There was an effort to eliminate barriers to creativity, creating a suitable atmosphere that encouraged adequate attitudes to develop creative skills. Results show that creative programs in schools, adapted to students' characteristics, will promote an increase in creative skills. 
Todos somos criaturas y creadores, pero diferimos en nuestra calidad de criatura $\mathrm{y}$ en nuestro poder de creación (Barron, 1969).

Podemos decir que el ser humano es creador por naturaleza, aunque, como dice Barron (1969), diferimos en nuestro poder de creación.

El campo de la creatividad quizás sea uno de los campos más atractivos del mundo de la psicología, y, al mismo tiempo, uno de los más ambiguos. Todo el mundo habla de creatividad, pero no todos hablan de lo mismo.

Al no tener unos patrones claramente definidos, se plantean preguntas como las siguientes: ¿Quién determina qué y quién es creativo?, ¿qué rasgos son los que conforman a una persona creativa?, ¿qué factores sociales y/o ambientales influyen en la persona creativa?, ¿está la creatividad relacionada de alguna manera con el coeficiente intelectual (CI)?, ¿la creatividad es un rasgo de personalidad, un rasgo cognitivo o ninguno de los dos? Éstas y otras preguntas se hacen desde el campo de la psicología sin llegar a obtener respuestas, en todos los casos, todo lo claras que se pretende.

Se puede sostener que así como se pueden desarrollar las capacidades de inteligencia, lo mismo podría decirse de las capacidades de creatividad.

Los programas de educación procuran realizarse pensando en el desarrollo de capacidades intelectuales, pero raras veces lo hacen pensando también en el de la creatividad. No sólo la inteligencia de las personas es necesaria para que avance el mundo, sino que es esa inteligencia unida a ciertas capacidades de creatividad la que hace que se produzcan descubrimientos como la teoría de la relatividad de Einstein.

\section{FUNDAMENTACIÓN TEÓRICA}

Como dice Davis (1981): "El pensamiento creativo es mucho más que utilizar la imaginación para obtener muchas ideas". Debe ser algo nuevo, original y adaptado a la realidad. Nuevo, en alguna de sus características, de tal forma que lo haga distinto de los otros, siendo cuando menos, infrecuente. Toda persona, con habilidades cognitivas normales, puede ser creativa en alguna medida y en alguna actividad en concreto.

Para desarrollar la creatividad son esenciales la educación y el entrenamiento en destrezas cognitivas. Las personas consideradas creativas no lo son siempre, ni tampoco en todos los campos. Hay ciertas características de la persona que parecen influir en la conducta creativa, como son: los intereses innatos, el talento, las destrezas cognitivas, la educación y la disposición personal

Los estudios de creatividad se han centrado, esencialmente, en la creatividad como producto, como proceso, como persona creativa, y han estudiado asimismo el medio y la situación en que se dan. 


\section{Producto creativo}

Como características básicas del producto creativo, hay que destacar la novedad y la adecuación. La novedad porque sea impredecible y la adecuación porque debe ser útil.

Para algunos autores, como Amabile (1983), la definición debe estar basada en la creatividad como producto, ya que el pensamiento creativo debe resultar en un fruto, el producto creativo. El producto será creativo en la medida en que los observadores apropiados así lo estimen. En general, las personas son capaces de determinar si un producto es creativo o no, a pesar de que no sean capaces de definirlo. Los especialistas en la materia del producto serán los encargados de decir en qué medida es creativo.
Amabile (1983) establece tres destrezas cognitivas como componentes de la producción creativa, como se resumen en la tabla $\mathrm{N}^{\circ} 1$.

Un aumento en las destrezas relevantes en un dominio, puede llevar a un aumento de creatividad. Las destrezas relevantes en creatividad determinan los distintos caminos por los que se puede llegar a la solución de un problema. Y la motivación hacia la tarea es la que principalmente va a marcar las diferencias entre lo que un sujeto puede llegar a hacer y lo que realmente hace.

Así, diremos que un producto será creativo en la medida en que sea nuevo, original, apropiado y así lo consideren los observadores expertos en esa materia.

Tabla $N^{\circ} 1$

\section{Componentes de la producción creativa según Amabile (1983)}

\begin{tabular}{lll}
\hline $\begin{array}{c}\text { Destrezas relevantes } \\
\text { en un dominio }\end{array}$ & $\begin{array}{c}\text { Destrezas relevantes } \\
\text { en creatividad }\end{array}$ & $\begin{array}{c}\text { Motivación hacia } \\
\text { la tarea }\end{array}$ \\
\hline $\begin{array}{c}\text { Incluye: } \\
\text { Conocimientos en un } \\
\text { dominio dado. }\end{array}$ & $\begin{array}{c}\text { Incluye: } \\
\text { Estilo cognitivo apropiado. }\end{array}$ & $\begin{array}{c}\text { Incluye: } \\
\text { Actitudes hacia la tarea. }\end{array}$ \\
Destrezas técnicas. & Conocimientos heurísticos & $\begin{array}{c}\text { Percepción de la propia } \\
\text { Talento especial en ese }\end{array}$ \\
dominio. & para generar ideas nuevas. & motivación para acometer la \\
& tarea. \\
Deptilo de trabajo adecuado. & \\
Habilidades cognitivas, & Depende de: & Depende de: \\
perceptuales y motóricas & Entrenamiento. & Nivel inicial de motivación \\
innatas. & Experiencia en la gene- & intrínseca hacia la tarea. \\
Educación formal o informal. & ración de ideas. & Presencia o ausencia de \\
& Características de & inhibidores sociales externos. \\
& personalidad. & Habilidades individuales para \\
& & minimizar cognitivamente los \\
inhibidores externos.
\end{tabular}




\section{Proceso creativo}

El producto creativo es el resultado de un proceso y la creación al ser un proceso puede desarrollarse.

Amabile (1983) presenta el proceso creativo en cinco etapas:

- Presentación.- El sujeto recoge la información referente al problema que tiene que resolver. En este punto es muy importante la motivación, ya que si la motivación intrínseca del sujeto es alta, será suficiente para afrontar el problema.

- Preparación.- Se trata de poner en marcha el almacén de información para preparar la posible solución al problema.

- Generación de respuestas.- Las respuestas que se van generando, deben ser novedosas. Aquí influirán, tanto la motivación a la tarea, como las distintas destrezas creativas que tenga el sujeto.

- Validación.- En esta etapa hay que validar la respuesta elegida como solución al problema. Hay que valorar si es válida y adecuada a la realidad del mismo.

- Aplicación y toma de decisiones.La decisión que se ha tomado en la etapa anterior hay que llevarla a la práctica. Una vez que se haya comprobado si es exitosa o no, termina el proceso. En caso positivo se aplicará y, en caso negativo, el problema se vuelve a situar en la primera etapa y la información adquirida se añade a la que ya se tenía. Hay que tener en cuenta que una tarea puede, a su vez, tener subtareas, que se situarán jerárquicamente. El éxito de la tarea dependerá de los resultados obtenidos en la solución de las subtareas.

Las personas, al afrontar los problemas, lo hacen de una manera divergente o convergente. A la hora de entrenar en creatividad se siguen modelos similares a los empleados en la resolución convergente de problemas, pero poniendo el énfasis en los procesos divergentes más que en los convergentes. En el entrenamiento de la creatividad, se espera que la actitud hacia ésta sea favorable, sin bloqueos emocionales, con gran originalidad, flexibilidad y fluidez.

\section{Persona creativa}

De la relación existente entre inteligencia y creatividad se han derivado, principalmente, dos cuestiones: la primera, que ambas están relacionadas entre sí; y, la segunda, que no tienen nada que ver la una con la otra, que son independientes. Estudios experimentales distintos apoyan las dos teorías. Lo que sí parece cierto es que la relación existente entre ellas es bastante compleja. La hipótesis que más se ha confirmado es que para que se dé la creatividad se tienen que dar unos valores de inteligencia mínimos, por encima del promedio. Es decir, de no superarse un cierto umbral de inteligencia es poco probable que ocurran altos niveles de 
creatividad. En consecuencia, la principal característica de la creatividad es la combinación de atributos intelectuales, estilo cognitivo y personalidad.

\section{El medio y la situación}

Un aspecto de la creatividad, que se ha ignorado durante bastante tiempo, ha sido el de las variables socioambientales y su interacción con rasgos de personalidad y destrezas cognitivas en la producción de respuestas creativas. Las personas con una motivación extrínseca están más supeditadas a las personas que las rodean, mientras que las motivadas intrínsecamente no lo necesitan, por lo que es más difícil que se dé la creatividad en las primeras. Se ha estudiado cómo influyen las recompensas económicas (motivación extrínseca) en el desarrollo creativo, llegando a la conclusión de que hay una relación inversa entre la motivación extrínseca (recompensa económica) y la creatividad.

La influencia del ambiente en la persona creativa depende, sobre todo, de factores de personalidad y situacionales. Hay ambientes laborales en los que la persona creativa es molesta para los superiores, se prefiere a la persona conformista, que no piense, que sólo trabaje. En ocasiones, pasa de forma similar en la escuela.

Vemos cómo la sociedad pone trabas a la creatividad, la inhibe y no la favorece, al menos, en muchas ocasiones. Se puede favorecer mucho la creativi- dad con sólo no impedir que se realice, reforzándola, creando planes de trabajo específicos para desarrollarla.

En la escuela se van haciendo cosas para favorecer el desarrollo de la creatividad, ejercicios tipo el proyecto $\mathrm{Eu}-$ reka, en el que se plantea un problema que el alumno tiene que resolver, de forma inteligente y creativa, trabajos abiertos en los que pueden llegar a la solución de distintas maneras. En algunas empresas se premian las ideas creativas que pueden mejorar los sistemas de trabajo, no obstante, esto está dando resultados contradictorios por lo comentado anteriormente en referencia a la motivación extrínseca.

Una teoría completa de creatividad será la que tenga en cuenta tanto los entornos como las personas.

\section{Potencial de creatividad}

Parece demostrado que se necesita un mínimo de CI para que se puedan desarrollar habilidades creativas en las personas. Pero no todas las personas con un CI elevado demuestran esas habilidades creativas. No obstante, toda persona es creadora, en mayor o menor medida y en una u otra tarea.

Por inteligente que sea una persona, puede mejorar de modo sustancial su alcance intelectual dirigiendo, de modo apropiado, sus pensamientos. Un individuo algo menos inteligente, que dispone de buenas habilidades para pensar, puede muy bien superar a una persona más inteligente que carece de estas habilidades, 
afirman Nickerson, et al. (1998). Estas habilidades debemos desarrollarlas mediante programas especializados, de tal suerte que toda persona pueda ser, en mayor o menor medida, más creativa. Al estimular las habilidades creativas, el sujeto puede aprovecharse de la gran cantidad de información que, sin ser consciente de ello, tiene almacenada en su cerebro. Por tanto, en toda persona, con unas habilidades cognitivas normales, existe un potencial creativo. Se trata de desarrollar ese potencial mediante estrategias creativas.

Otra característica a tener en cuenta para desarrollar el potencial de creatividad son los obstáculos a la creatividad. En la mayoría de las personas existen tres tipos de bloqueos que inhiben el desarrollo de la creatividad; bloqueo perceptual, cultural y emocional. "Partiendo de la base de que el conocimiento de las barreras a la creatividad permite aumentar el potencial creativo..." (Davis, 1992), sería conveniente que en todo programa de desarrollo de la creatividad, los sujetos conociesen previamente sus obstáculos a la creatividad.

Siete puntos nos parecen especialmente importantes para desarrollar el potencial de creatividad en los sujetos:

- Estimular las actitudes favorables hacia la creatividad.

- Eliminar las barreras a la creatividad.

- Crear el clima adecuado para el desarrollo de la creatividad.
- Fomentar estilos cognitivos favorecedores del desarrollo de la creatividad.

- Utilización adecuada de los recursos que tiene el sujeto.

- Enseñar estrategias para el desarrollo de habilidades creativas.

- Reforzar las situaciones creativas.

\section{Programa de intervención para el} DESARROLLO DE LA CREATIVIDAD

El programa de intervención que hemos desarrollado está basado en las cinco etapas del proceso creativo de Amabile (1983), descrito con anterioridad.

En cada sesión se dan nuevas estrategias para que puedan aplicarlas en la resolución de los problemas que se plantean. Así mismo, al final de cada sesión se realiza una puesta en común en la que cada sujeto explica el proceso creativo que ha seguido para llegar a la solución del problema. Esto sirve de enriquecimiento para todos porque conocen las estrategias seguidas por los otros sujetos, al tiempo que se refuerzan los procesos seguidos.

\section{Estructura del programa}

- Charla explicativa sobre la creatividad y sobre el diseño del programa al grupo de alumnos que se va aplicar el programa. El conocimiento del tema y del diseño es muy importante para su nivel de motivación y participación adecuada. 
- Aplicación de una prueba inicial para conocer el nivel creativo de los sujetos al inicio del programa.

- Aplicación de un inventario de barreras del pensamiento creativo, porque conocer sus propias barreras a la creatividad será un primer paso para aumentar el potencial de creatividad.

- Seguidamente se aplican de cinco a diez sesiones, con arreglo a las siete etapas siguientes:

- Antes de comenzar cada sesión se explican las nuevas estrategias para el desarrollo de la creatividad y aumento de destrezas creativas por parte del sujeto.

- Presentación de un problema. Son problemas que requieren mejoras o soluciones nuevas.

- Los problemas deberán estar adaptados a las capacidades y nivel de instrucción de los alumnos.

- Preparación, por parte del sujeto, de las posibles soluciones. El sujeto recaba información de su propio almacén y pone en práctica las destrezas que se le han explicado anteriormente.

- Generación de respuestas. El sujeto da la solución al problema de forma gráfica y explicativa en una hoja de respuestas.

- Seguidamente se hace una puesta en común en la que cada uno expone la solución a la que ha llegado y, sobre todo, el proceso creativo que ha seguido para darle solución al problema. Se hacen y contestan todas las preguntas necesarias para aclarar dicho proceso. Sirve de refuerzo $\mathrm{y}$ todos se enriquecen de todos.

- A continuación se realiza la validación. Un grupo de expertos en los problemas planteados valora la validez y adecuación de las respuestas.

- Aplicación y toma de decisiones. La decisión tomada en la etapa anterior se lleva a la práctica. Una vez comprobada su aplicación, termina el proceso. En caso positivo se aplica $y$, en caso negativo, el problema se vuelve a situar en la primera etapa y la información adquirida se añade a la que ya se tenía (realimentación). Si no es posible la aplicación práctica, el grupo de expertos decide la viabilidad práctica del producto, dándole al sujeto la información correspondiente que le sirva de realimentación y refuerzo.

- Aplicación de una prueba final para conocer las variaciones en capacidad creativa debidas al programa que se ha aplicado, tomando como referencia los valores que obtuvieron en la prueba inicial.

\section{Implementación del programa}

El programa se aplicó a diez sujetos de un grupo de estudiantes de segundo curso de la carrera de diseño industrial. 
Para una buena adaptación del programa a las características del grupo, se partió del conocimiento de la carrera y del grupo de alumnos al que se iba a aplicar. La principal ventaja de realizar la intervención en un grupo de estudiantes de diseño industrial es que la motivación hacia temas de desarrollo de creatividad es alta. El mayor inconveniente es que los resultados pudieran estar influenciados por los propios trabajos de diseño que realizan durante el curso y que les sirven de entrenamiento en el desarrollo de la creatividad. Para controlar el aprendizaje no debido a la intervención del programa, se hicieron dos grupos: experimental y control, y el programa se desarrolló en período no lectivo.

- Objetivo: Diseñar, aplicar y constatar un programa de intervención para el desarrollo de la creatividad.

- Hipótesis: Si a un grupo de sujetos bien motivados le aplicamos un programa de intervención para el desarrollo de la creatividad, adecuado a las características del grupo, se producirá en ellos un incremento de ésta.

A continuación se detallan las siete sesiones en las que se realizó la intervención, siguiendo la estructura desarrollada anteriormente.

\section{Primera sesión}

a) Charla explicativa a un grupo de alumnos de segundo curso de la carrera de diseño industrial, sobre la creatividad y el programa elaborado para desarrollarla.

b) Aplicación de la forma A (cuatro primeros juegos) del test de P. Torrance, como prueba inicial para conocer su nivel de desarrollo creativo al inicio del programa.

c) Aplicación del inventario de barreras del pensamiento creativo y de la acción innovadora de Lorna P. Martin (1990).

d) Asignación aleatoria de sujetos a los grupos experimental y control. Cinco sujetos a cada grupo.

Las sesiones 2, 3, 4, 5 y 6 siguientes se realizan, únicamente, con el grupo experimental.

\section{Segunda sesión}

a) Nuevas estrategias.- Se dan los resultados del inventario de barreras del pensamiento creativo y de la acción innovadora realizada en la sesión anterior, así como una explicación oral y una hoja explicativa de los resultados. Esto servirá de motivación para que, una vez conocidas las barreras a la creatividad que cada uno tiene, les sea más fácil superarlas en situaciones sucesivas y obtener así un mayor potencial creativo.

b) Presentación de un problema.- Diseño de un paquete de tabaco con cerillas incorporadas en éste. Son problemas relacionados con la carrera, que requieren mejoras o soluciones nuevas. 
c) Preparación.- El sujeto recaba información de su propio almacén, procurando salvar las barreras a la creatividad vistas anteriormente.

d) Generación de respuestas.- En la hoja de respuestas el sujeto dibuja y explica el diseño. También deberá tener en cuenta el coste económico aproximado del producto (viabilidad práctica), dado que es la manera como se trabaja en la carrera y en su futura vida profesional.

e) Puesta en común.- Cada uno expone la solución a la que ha llegado, explicando el proceso creativo que ha seguido hasta dar con la solución al problema. Se establece un diálogo que ayude a aclarar el proceso seguido, así como la viabilidad y utilidad del producto diseñado.

f) Validación.- Un grupo de expertos del diseño industrial valora la utilidad, originalidad y diseño del producto realizado por cada uno de los sujetos. Las puntuaciones oscilan entre 1 y 5 puntos, recibiendo 1 los trabajos muy malos y 5 los muy buenos.

g) Aplicación y toma de decisiones.El grupo de expertos del diseño industrial decide la viabilidad práctica del producto en cada caso, valorando de 1 a 5 puntos, como en el apartado anterior, cada uno de los diseños, teniendo en cuenta las características propias del producto y del mercado. Las valoraciones dadas en estos dos apartados sirven de reali- mentación y refuerzo a los sujetos y les ayudan a mejorar en las siguientes sesiones.

\section{Tercera sesión}

a) Nuevas estrategias.- Explicación general de las estrategias y técnicas del desarrollo del proceso creativo. Se explica más explícitamente "la tormenta de ideas" (brainstorming), haciendo hincapié en la utilización de esta técnica en la resolución del problema que se les va a plantear a continuación.

b) Presentación de un problema.- Papelera para recreo de colegio de educación primaria y secundaria; y

c) Preparación.- El sujeto recaba información de su propio almacén, procurando aplicar, especialmente, la técnica "tormenta de ideas" (brainstorming).

Los apartados d, e, fy g se realizan de la misma forma que en la segunda sesión.

\section{Cuarta sesión}

a) Nuevas estrategias.- Explicación de la técnica Listas de comprobación (idea checklist). A continuación, cada sujeto realiza una "lista de comprobación" y posteriormente se hace una puesta en común para que todos amplíen la lista que ya tenían.

b) Presentación de un problema.- Diseño de una caja de ordenador con los siguientes elementos: disco du- 
ro, disquetera de $3.5, \mathrm{CD}$, grabador de CD, ZIP, DVD, interruptor, pulsador turbo y pulsador reset.

c) Preparación.- Trabajando con la lista confeccionada anteriormente deben resolver el problema que se les ha planteado.

Los apartados d, e, f y g se realizan de la misma forma que en la segunda sesión.

\section{Quinta sesión}

a) Nuevas estrategias.- Explicación de la técnica "lista de atributos" (attribute testing).

b) Presentación de un problema.- Diseño de un lavabo. A continuación cada sujeto realiza una "lista de atributos" relacionada con el problema. Posteriormente, se hace una puesta en común y cada sujeto amplía su lista con las aportaciones de los otros sujetos.

c) Preparación.- Trabajando con la lista realizada anteriormente deben resolver el problema.

Los apartados d, e, fy g se realizan de la misma forma que en la segunda sesión.

\section{Sexta sesión}

a) Nuevas estrategias.- Explicación de la técnica "la sinéctica" (synectics).

b) Presentación de un problema.- Cenicero con pie.

c) Preparación.- Igual que en sesiones anteriores, deben utilizar la técnica que se les ha explicado para la resolución del problema planteado.

Los apartados d, e, f y g se realizan de la misma forma que en la segunda sesión.

\section{Séptima sesión}

Aplicación de la forma A (juegos cinco, seis y siete) del test de P. Torrance a los dos grupos (experimental y de control), como prueba final para conocer las variaciones en capacidad creativa debidas al programa que se ha aplicado. Se toman como referencia los valores de la prueba inicial y los valores del grupo de control.

\section{RESUltados}

Hemos realizado un valor total único en creatividad con las puntuaciones de cada sujeto y con las de cada grupo en conjunto, tanto de la prueba inicial como de la prueba final, tomando la media de los tres procesos del test de Torrance (fluidez, flexibilidad y originalidad). De forma resumida se presentan los datos en la tabla $\mathrm{N}^{\circ} 2$. Estos valores totales de creatividad son los que se han comparado entre sí para comprobar las variaciones en creatividad en el grupo experimental, y entre el grupo experimental y el grupo control.

El análisis de los datos lo realizamos a nivel individual y a nivel de grupo y, fundamentalmente, en tres apartados: primero, en el grupo experimental; segundo, en el grupo control, y tercero, 
Tabla No 2

Valores de creatividad de los grupos experimental y control

\begin{tabular}{cccccc}
\hline \multicolumn{3}{c}{ Grupo experimental } & & Grupo control \\
Prueba & Prueba \\
inicial & final & Sujetos & $\begin{array}{c}\text { Prueba } \\
\text { inicial }\end{array}$ & $\begin{array}{c}\text { Pruebl } \\
\text { final }\end{array}$ \\
\hline 1 & 14 & 16 & 1 & 14 & 15 \\
2 & 8 & 10 & 2 & 11 & 13 \\
3 & 11,5 & 20 & 3 & 10,5 & 10 \\
4 & 11 & 18 & 4 & 8 & 6 \\
5 & 14 & 20 & 5 & 12 & 11 \\
Total & 11,7 & 16.8 & Total & 11,1 & 11 \\
\hline
\end{tabular}

entre los grupos experimental y control. En los dos primeros nos hacemos las siguientes preguntas: ¿ha habido variaciones en los valores de creatividad en los sujetos entre la prueba inicial y la prueba final?, ¿ha habido variaciones en los valores de creatividad en el conjunto del grupo entre la prueba inicial y la prueba final? En el tercer caso nos planteamos si hay diferencias entre los dos grupos y, en el caso de haberlas, si son significativas.
$\mathrm{A} 1$ inicio del programa los valores en creatividad de los dos grupos son bastante similares, lo que demuestra la igualdad entre ambos grupos.

En los valores obtenidos por el grupo experimental podemos comprobar que todos los sujetos han aumentado en creatividad en la prueba final en relación con la prueba inicial (figura $\mathrm{N}^{\mathrm{o}} 1$ ), el aumento, lógicamente, no es el mismo en todos los sujetos debido a las diferencias individuales existentes entre

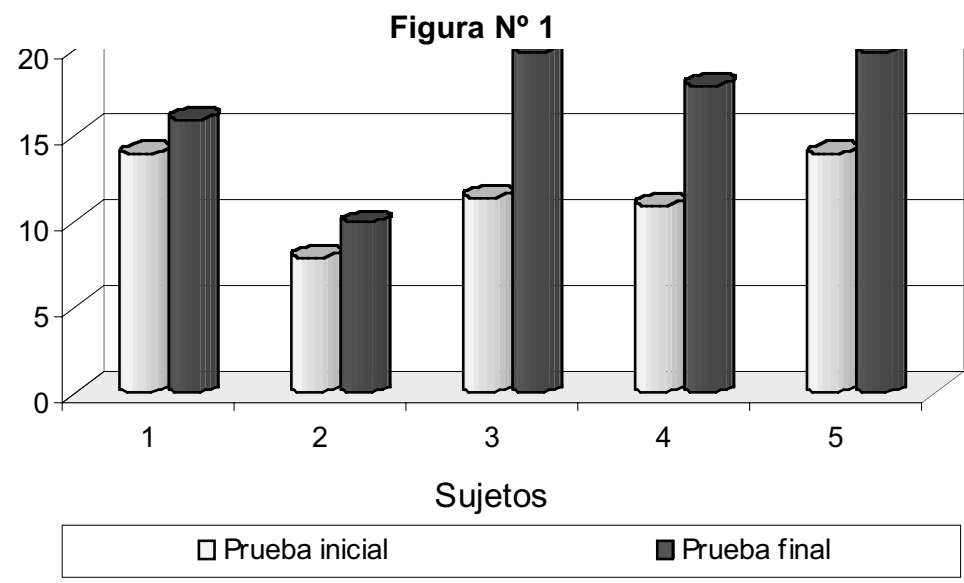




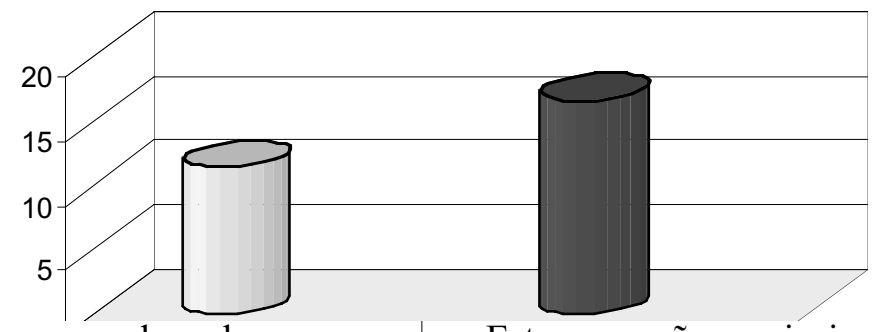

ellos. De forma general, podemos ver

Estas pequeñas variaciones son lógieste aumento en creatividad debido al cas dado que las pruebas inicial y final programa, en los resultados totales de grupo de la prueba inicial y la prueba final (figura $\mathrm{N}^{\circ} 2$ ).

En los datos obtenidos del grupo control observamos pequeñas variaciones entre los valores de la prueba inicial y los de la prueba final (figura $\mathrm{N}^{\mathrm{o}} 3$ ).

se realizaron con seis semanas de diferencia y en ese tiempo no tuvieron entrenamiento específico en creatividad al no realizar el programa y no ser periodo lectivo de clases.

De forma general, comprobamos en los resultados totales de grupo como

Figura $\mathbf{N}^{\circ} 3$

Valores de creatividad del grupo control

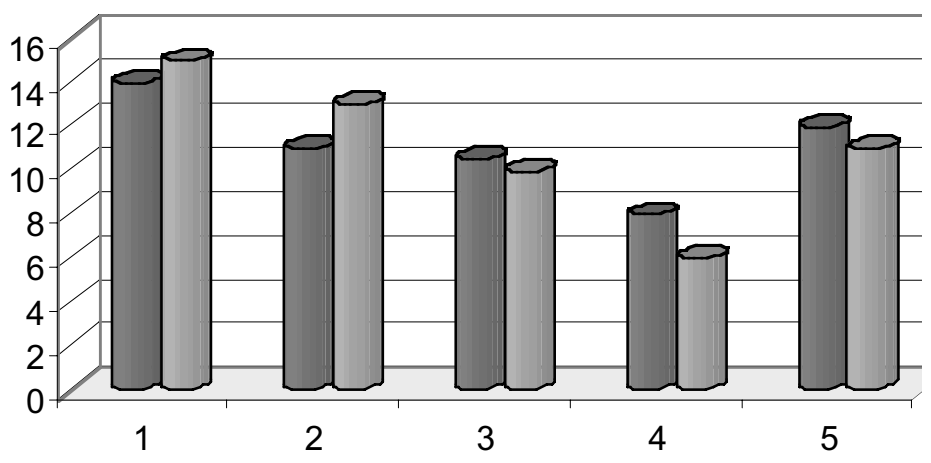


los valores de la prueba inicial y la prueba final quedan muy igualados (figura $\mathrm{N}^{\circ} 4$ ), demostrando que los cambios producidos en el grupo experimental son debidos al programa de intervención.

Al comparar los resultados del grupo experimental con los del grupo control (figura $\mathrm{N}^{0}$ 5) se aprecia cómo en la prueba inicial no hay diferencias significativas entre ambos grupos, estando bastante igualados al comienzo de la implementación del programa y, sin embargo, en los valores de la prueba final se aprecian diferencias significativas entre ambos debido al incremento de los valores

Figura $\mathbf{N}^{\circ} 4$

Valores totales de creatividad del grupo control

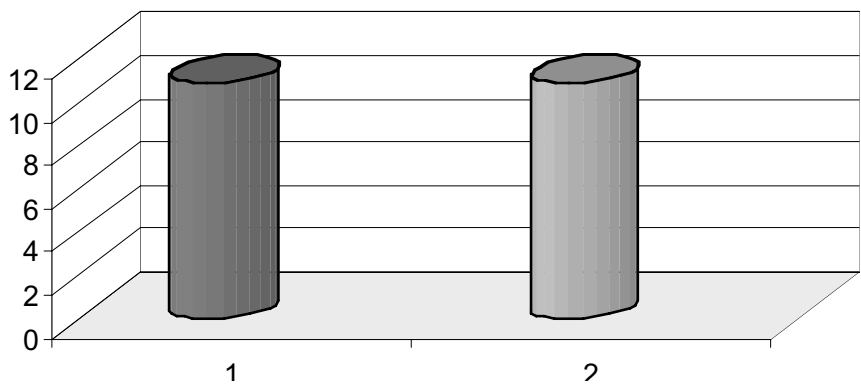

Figura $\mathrm{N}^{\circ} 5$

Valores totales de creatividad

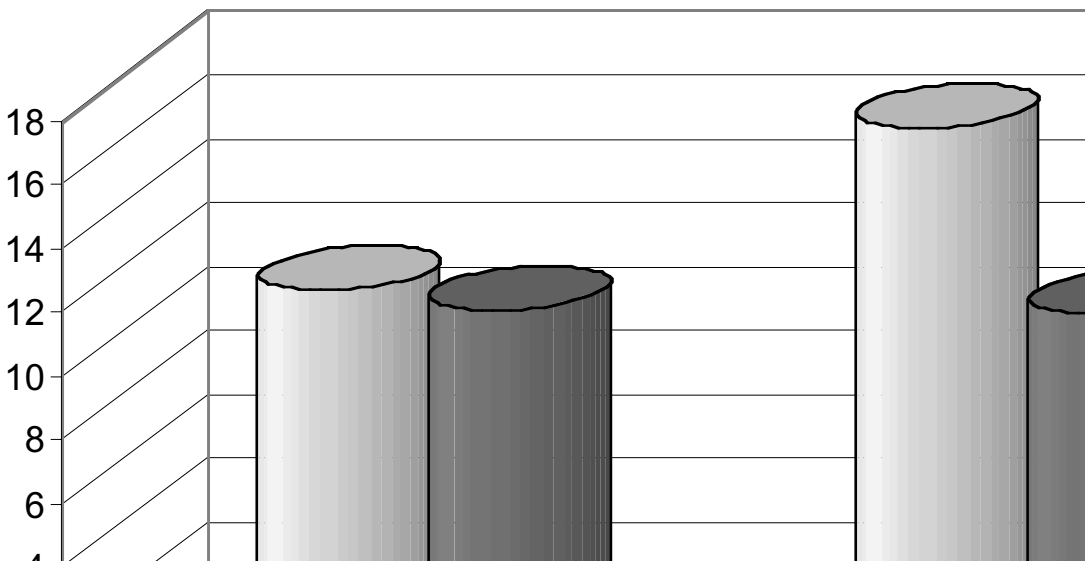


de creatividad del grupo experimental. No podemos decir que este incremento sea debido a un efecto de maduración, dado que nos sirve de referencia el grupo control, así mismo, no es debido al desarrollo producido por las materias tratadas en clase, ya que el programa se implementó en periodo no lectivo.

\section{DISCUSIÓN}

Por el número reducido de la muestra de los dos grupos y por el corto periodo de tiempo en el que se ha aplicado el programa, no podemos decir que los resultados sean concluyentes, aunque sí podemos decir que son muy alentadores, dado que demuestran que se puede conseguir mucho si se trabaja el desarrollo de la creatividad en los colegios con programas especialmente diseñados para las características propias de los alumnos de cada nivel.

En el programa se ha procurado incidir en los siete puntos que consideramos importantes para el desarrollo del potencial creativo comentados con anterioridad.

Las principales características del programa que han incidido de forma especial en el aumento de la creatividad son:

- El conocimiento, por parte de los sujetos, de sus barreras a la creatividad desde el inicio del programa.

- La alta motivación hacia el programa, dado que al ser estudiantes de diseño industrial son conscientes de la importancia que tiene la creatividad en la profesión elegida.
- La explicación y aplicación de nuevas estrategias para el desarrollo de la creatividad en cada sesión, y la realización de una práctica (adecuada al estilo de diseños que realizan en la carrera) utilizando especialmente la estrategia explicada en esa sesión.

- La puesta en común en la que cada uno expone su proceso creativo en la resolución del problema, así como las preguntas que se suscitan para ayudarle a conocer y dar a conocer mejor el proceso que ha seguido, enriquecen a todos, en primer lugar porque fortalecen las técnicas empleadas adecuadamente, y en segundo lugar porque descubren nuevos procesos seguidos por los compañeros.

- La fase de validación, realizada por los expertos de la carrera, les servía de retroalimentación y esfuerzo al comprobar la aplicación de las estrategias de creatividad aprendidas al mundo del diseño industrial.

La sociedad se preocupa bastante en medir la inteligencia y, sin embargo, los principales esfuerzos y avances parecen venir de la creatividad. Esa originalidad y adaptación que da la creatividad es la que marca la diferencia entre una persona mediocre y otra excepcional.

Para desarrollar la creatividad se debe tener en cuenta la combinación de atributos intelectuales, el estilo cognitivo, la personalidad y las estrategias. Igualmente, es importante eliminar las ba- 
rreras a la creatividad, crear un clima adecuado y estimular actitudes favorables que ayuden a desarrollar las habilidades creativas.

En la medida de lo posible será conveniente aplicar los programas en el medio natural del aula, adaptándolos a las materias del curso, de tal forma que el alumno desarrolle la creatividad en el trabajo diario de clase como algo natural.

\section{REFERENCIAS}

Amabile, T.M. (1983). The social psychology of creativity: A componential conceptualization. Journal of Personality and Social Psychology 45 (2), 357-376.

Barron, F. (1969). Creative person and creative process. New York: Holt, Rinehart \& Winston.

Boden, M.A. (1994). La mente creativa. Mitos y mecanismos. Barcelona: Gedisa.

Davis, G.A. \& Scott, J.A. (1992). Estrategias para la creatividad. Buenos Aires: Paidós.

De Sánchez, Margarita A. (1995). Desarrollo de habilidades del pensamiento. Creatividad. México: Trillas.

De Sánchez, Margarita A. (1995). Desarrollo de habilidades del pensamiento. Creatividad. Guía del instructor. México: Trillas.

Fustier, M. (1993). Pedagogía de la creatividad. Madrid: Editorial Index.

Nickerson, R.S.; Perkins, D.N.; \& Smith, E.E. (1998). Enseñar a pensar. Madrid: Paidós/M.E.C. 